\title{
MedienPädagogik
}

Zeitschrift für Theorie und Praxis der Medienbildung

www.medienpaed.com

ISSN 1424-3636

Themenheft Nr. 37: Medienpädagogik als Schlüsseldisziplin in einer mediatisierten Welt. Perspektiven aus Theorie, Empirie und Praxis Herausgegeben von Henrike Friedrichs-Liesenkötter, Lara Gerhardts, Anna-Maria Kamin und Sonja Kröger

\section{Der Wert der Medien in medienpädagogischen Biografien}

Christian Swertz

\section{Zusammenfassung}

In biografischen Erzählungen weisen sich Menschen selbst einen Wert zu. Das ist bei Wissenschaftlerinnen und Wissenschaftlern nicht anders. Eine Form solcher Erzählungen sind Erzählungen der Geschichte der Wissenschaft, in denen Wissenschaftlerinnen und Wissenschaftler der Wissenschaft und damit sich selbst einen Wert zuweisen. Es wird gezeigt, dass Medien in solchen Erzählungen ein Wert für die Medienpädagogik gegeben wird und die Medienpädagogik gleichzeitig an der Erzeugung des Werts beteiligt ist. Die Produktion des Werts von Medien und der Medienpädagogik durch Medienpädagoginnen und Medienpädagogen wird abschliessend werttheoretisch reflektiert.

\section{The value of media in biographies of media educationlists}

\begin{abstract}
In biographies people assign themselves a value. That applies to scientists as well. One form of such stories are stories about the history of a scientific field where scientists assign a value to the scientific field and thus to themselves. It is shown that a value is given to media in stories about media education and media education contributes to the value of media. The production of the value of media and media education by media educators is reflected with the theory of value.
\end{abstract}




\section{Einleitung}

Die meisten Wissenschaftlerinnen und Wissenschaftler finden es schön, ihre Aufsätze als Heldinnen- und Heldenreise und in der Dramaturgie einer Drei-Akt-Struktur aufzubauen: Der erste Akt besteht aus der einleitenden Darstellung des Forschungsstandes mit dem Forschungsproblem als Herausforderung, das die Heldinnen und Helden verletzt, weil Unwissenheit droht. Das führt zur Forschungsfrage als erstem Plotpoint - wenn die mal gestellt ist, gibt es keinen Weg mehr zurück. Im Hauptteil folgt dann die Suche nach dem Lösungsweg (Akt 2a - Methodendiskussion), in der die Antagonisten (Methodenprobleme) überwunden werden. Ist der Lösungsweg gefunden, kann die Herausforderung auf dem Weg zum zweiten Plotpoint gezielt angegangen werden (Akt 2b - Ergebniskapitel). Der zweite Plotpoint liefert den Höhepunkt mit der Überwindung der zentralen $\mathrm{He}$ rausforderung. Im Showdown (Diskussion) wird die Herausforderung bewältigt, indem die Heldinnnen und Helden sich zu Wissenden entwickeln. Das ist eine veritable Charakterentwicklung.

Eine Eigenart des Subgenres der wissenschaftlichen Heldenreise ist, dass die Heldinnen und Helden kaum auftreten, sondern sich in ihren Taten zu erkennen geben. Verwendet wird meist eine auktoriale Erzählsituation mit den Heldinnen und Helden als Erzählerinnen und Erzählern. Es ist vermutlich dieser Umstand, der das Format so attraktiv macht.

Ein unverzichtbares Element des Genres sind Daten. Alle Wissenschaftlerinnen und Wissenschaftler müssen Daten so lesen, dass eine Verständigung über die Interpretation der Daten hergestellt werden kann. Insofern daher jede Wissenschaft darin besteht, sich im Verhältnis zur Welt, zu sich selbst und zu anderen zu entwickeln (Meder 2017), sind alle Wissenschaften auf das Bildungsproblem verwiesen.

Dabei geht es in Wissenschaft und Bildung seit einiger Zeit nicht nur um diese Verhältnisse, sondern auch um das Verhältnis zu diesen Verhältnissen (Meder 2015). Für wissenschaftliches Handeln entscheidend wird der Umstand, um die Verhältnisse zu wissen und dies explizit zu schreiben - eine Struktur, die in den typischen Merkmalen des Genres «Wissenschaftlicher Aufsatz» zum Ausdruck kommt.

Reflexivität ist auch im Blick auf die Geschichte der Gemeinschaft nötig, denn bei den Verhältnissen handelt es sich nicht um lineare Mechanismen, 
sondern um korrelative Beziehungen. Daher sind Aussagen mit wissenschaftlichem Wahrheitsanspruch weder unabhängig von der Geschichte der Gemeinschaft (Barberi 2000, 203ff.) noch gehen sie darin auf: Wahrheit ist korrelativ auf die Angemessenheit der Methode für den Gegenstand und die Tradierung von Geltungsbeständen bezogen (Hönigswald 1927).

Dieses Problem wird mit der Korrelation von Lebenslauf (Daten) und Biografie (Interpretationen) zum Ausgangspunkt biografischer Forschung; eine Verwicklung, die in der Biografieforschung entwickelt wird, wenn auch nicht reflexiv: Die Verwicklung der eigenen Biografie in die wissenschaftlichen Lese- und Schreibprozesse wird, anders als in anderen Ansätzen (Borkert u.a. 2006), in biografischen Studien (z.B. bei Metz 2016) wenig bedacht. Zudem schliessen die etablierten Riten biografischer Forschung multiple Medienreflexionen ein (Orale Erzählung, digitale Aufzeichnung, Rezeption mit multiplen Medienreflexionen in der Transkription etc.), die weder harmlos sind noch reflektiert werden und daher geradezu wirksam werden können.

Während die medialen Implikationen biografischer Forschung wenig thematisiert werden, werden die biografischen Implikationen von Medien in der medienbiografischen Forschung spätestens seit Mitte der 1970er Jahre untersucht (Rogge 1980; Hickethier 1980). Dabei wird dieser Ansatz gelegentlich mit einer Wendung gegen «den Mainstream der Medienforschung» (Aufenanger 1999: 488) begründet, womit biografische Ansätze in eine exkludierte Position gebracht werden. Diese Positionierung ist allerdings weder in der medienbiografischen Praxis (Pöyskö 2009) noch in medienbiografischen Studien dominant. Betont wird vielmehr das Potential der medienbiografischen Perspektive zur Reflexion und Analyse des Medienhandelns in der Lebenswelt (Rogge 1982; Baacke 1983), wobei gelegentlich die biografische Perspektive nicht klar von der historischen Perspektive unterschieden wird (z.B. bei Buchen und Philipper 2003).

Die biografische Perspektive wird in medienbiografischen Studien häufig mit anderen Forschungsmethoden und -perspektiven verbunden, etwa mit der sozialökologischen Perspektive (Baacke u.a. 1990, 7). Das gilt vor allem für die Studien, die im Rahmen des metakommunikativen Ansatzes von Baacke (1973) durchgeführt wurden. Im Rahmen dieser Studien wurde z.B. herausgearbeitet, wie das Fernsehen Menschen mit Sinnangeboten 
versorgt, die als vielfältig und widersprüchlich beschrieben werden, was wieder in einen Zusammenhang mit der technischen Entwicklung gestellt wird (Meister 1997).

Dabei wird es noch 1990 als begründungsbedürftig gesehen, dass Medien eine biografische Relevanz besitzen (Baacke u.a. 1990, 10), was vermutlich vor allem durch den Umstand zu erklären ist, dass medienbiografische Daten, die bei Jugendlichen Ende der 1980er Jahre erhoben wurden, einen Zeitraum erfassen, in dem wenig technologische Veränderungen des im Alltag verfügbaren Medienensembles erfahrbar waren.

In der Analyse wenig berücksichtigt wurde der Umstand, dass die «tiefgreifenden Veränderungen vom Normalentwurf eines Lebenslaufs im Sinne von Destandardisierungen und Dechronologisierungen» (ebd., 12) sich nicht nur im medienbezogenen Handeln zeigen, sondern durch medienbezogenes Handeln zugleich produziert werden.

Herausgearbeitet wird aber, dass Medien Material «für eine eigene Weltkonstruktion» (ebd., 13) liefern und zugleich Orientierungsdilemmata (Meister 2007, 273) verstärken. Die Konfrontation von Menschen mit verschiedenen in Medien angebotenen Orientierungen kann allerdings ebenso wie die Konfrontation mit verschiedenen Sprachen (Humboldt 1997, 91) als Bildungsanlass verstanden werden, denn die nicht durch äussere Massgaben auflösbaren Dilemmata fordern dazu auf, sich selbst zu orientieren (Meister und Meise 2010, 197), d.h. sich kreativ eine eigene Gewissheit in Sprachspielen zu verschaffen (Meder 1986). Das gelernt zu haben, zeichnet einen gebildeten Menschen aus, was es erforderlich macht, die in Medienkompetenzbegriffen oft genannt kreative Gestaltung auch auf sich selbst zu wenden (Meister 2008, 525).

Der These der Destandardisierung wurde in einer Studie von Meister (1997b) die Beobachtung gegenübergestellt, dass «auffallende Parallelitäten in den medialen und konsumptiven Geschmacksstilen» (Meister 1997b, 190) bei jugendlichen Aussiedlerinnen und Aussiedlern aus Polen und den Jugendlichen der deutschen Aufnahmegesellschaft zu beobachten sind, wobei die übersiedelten Jugendlichen sich selbst durch die Medien zugleich als «Fremde» konstruiert sehen (ebd, 227).

Seit 1990 hat sich die mediale Inhalts- und Infrastruktur deutlich verändert. Damit wird z.B. eine Steigerung von «Reflexivität und Flexibilität» 
(Meister und Meise 2010) verbunden, allerdings weder auf Medienbiografien noch auf die Medienpädagogik als Wissenschaft selbst bezogen.

Nun ist anzunehmen, dass im Falle erheblicher Veränderungen in den Lebenswelten auch die Handlungen von Wissenschaftlerinnen und Wissenschaftlern betroffen sind - nicht nur durch gestiegene Erwartungen Studierender an institutionalisierte Bildungsangebote (Meister 2004), sondern auch durch die Veränderungen der Produktionsprozesse im Wissenschaftsbetrieb, in dem mit der Nutzung digitaler Technologien «das Arbeitsmittel aus einem Werkzeug in eine Maschine» verwandelt wurde, was zu einer Veränderung in den «Kommunikations- und Transportmitteln» führt, deren Herstellung in einem zyklischen Prozess «zyklopische Maschinen» (Marx 1962, 391ff.) erforderlich macht - wobei es gelungen ist, den Gebrauchswert des Tauschwerts der Arbeitskraft zumindest in der Lehre und der Anwendungsentwicklung zu erhalten. In der Grundlagenforschung wird dies zunehmend nur noch denjenigen, «die aussergewöhnliche wissenschaftliche Fähigkeiten zeigen» (Hayek 2005: 504) zugestanden, wobei nach Hayek «einem Kommunisten kein ,tenure' gegeben werden sollte» (ebd, 509), was eine ideologiekritische Analyse erspart und die aktuellen Untiefen der öffentlichen Medienkompetenzdiskussion, in der im Unterschied zur Fachdiskussion oft die ökonomische Nutzbarmachung von Menschen als einzige Massgabe genannt wird, markiert.

Medienbildung ist damit eine Aufforderung zur Selbstbildung an Wissenschaftlerinnen und Wissenschaftler im Blick auf die eigene Medienproduktion und die eigene Medienbiografie. Interessant ist in diesem Zusammenhang, dass die zwölf Phasen der Heldenreise nach Campbell keine Äusserlichkeiten sind, sondern, im Anschluss an Jung, Ausdruck eines Archetypen. Sie beschreiben die Entwicklung aller Menschen, die zu Heldinnen und Helden werden können, wenn sie ihrer inneren Stimme folgen - wobei Campbell mit der These, dass der Mythos «unverdorben von den Urquellen menschlichen Lebens und Denkens» (Campbell 1999, 26) kommt, die Machtförmigkeit seiner Konstruktionsleistung übergeht. Die Interpretation der Mythen wird von Campbell zu einem «Bildungsprogramm» gewendet, das sich als wachstumsorientiertes Selbsterfahrungstraining (Rebillot und Kay 2011) oder in der antroposophischen Biografiearbeit durchaus verkaufen lässt (Seyffer 2011) - wobei das unreflektierte Vertrauen auf 
die innere Stimme in beiden Fällen die Frage nach Werten und Interessen heraufbeschwört. Aber das ist ein anderes Thema. Wichtiger ist, dass die Heldenreise von Campbell wieder in einen Mythos gewendet wurde, was darauf aufmerksam macht, dass es durchaus relevant sein kann, das Genre des wissenschaftlichen Aufsatzes aus medienpädagogischer Sicht zu dekonstruieren sowie die persönliche Korrelation von Biografie und Interpretation zu bedenken.

\section{Methode}

Wenn Erzählungen immer auch von der Biografie der Erzählerinnen und Erzähler abhängen, gilt das auch für die Erzählung der Geschichte einer Wissenschaft. Insofern in dieser Positionalität die Biografie zum Ausdruck gebracht wird, erscheint es lohnend, Erzählungen der Geschichte der Medienpädagogik als autobiografische Erzählungen zu lesen, als thematisch umgrenzten Ausdruck der Rekonstruktion der eigenen Geschichtlichkeit.

Dabei ist zunächst zu berücksichtigen, dass sich die Geschichte der Medienpädagogik «vor dem Hintergrund der Entwicklung der Medientechnologie» (Schäfer 2001, 27) vollzieht. Dieser Hintergrund kann als Bestandteil des jeweiligen konjunktiven Erfahrungsraums (Schäffer 2005) beschrieben werden, der in dem hier untersuchten atypischen, nichtreaktiven und ökologisch validen Dokumenttyp, in dem kulturelle Muster befolgt werden, zum Ausdruck gebracht wird.

Die Rekonstruktion des konjunktiven Erfahrungsraums ist Ziel der dokumentarischen Methode. Mit dieser Methode können die relevanten Dokumente als autobiografische Texte gelesen werden. Allerdings erlaubt die dokumentarische Methode dabei nur einen eingeschränkten Blick, denn die Lektüre wissenschaftlicher Texte als autobiografische Texte in der Absicht der Formulierung eines wissenschaftlichen Textes impliziert eine spezifische Relationierung von Rezeption und Produktion, die in der Methodologie der dokumentarischen Methode wenig reflektiert wird. Eine weitere Grenze liegt in dem Umstand, dass die Untersuchung konjunktiver Erfahrungsräume Zusammenhänge «des Geschlechts, der Generation oder der Migration» berücksichtigt (Fuchs 2011, 177), Werte und Interessen aber kaum in den Blick nimmt. Zwar wird mit der Berücksichtigung 
des Kontextes von Biografien (Alheit und von Felden 2009) die Korrelation von Selbst und Anderen berücksichtigt, mit der das Verhältnis von Herrschafts- und Machttechniken in den Blick genommen werden könnte. Das hat jedoch weder zu einer methodischen Selbstreflexion (Habermas 2001, 262) noch zur Desillusionierung (Wigger 2016, 126) geführt.

Am Experimentieren mit den Perspektiven hindert das nicht, weil Grenzen notwendig vorkommen, insofern es sich bei Letztbegründungen um Invarianten handelt, hinter die nicht argumentierend zurückgegriffen werden kann (Hönigswald 1926, 83). Daher sind Beobachtungen nur innerhalb eines begrifflich fundierten Rahmens sinnvoll (Swertz 2017a). Den zu entwickeln wird hier nicht beansprucht. Es geht mir hier nicht zuletzt unter dem Eindruck der aktuellen Produktionsbedingungen wissenschaftlichen Wissens nur um ein vorläufiges Spiel mit Perspektiven als Leser.

Auf diesem Weg einen Blick auf Texte zur Geschichte der Medienpädagogik zu werfen, wird hier als Wissenschaftspädagogik bezeichnet, weil die Positionen der Erzählerinnen und Erzähler und die darin zum Ausdruck gebrachte eigensinnige Identität im Sinne des «individuellen Allgemeinen» (Fuchs 2011, 277) in den Mittelpunkt gerückt wird. Dazu werden im Folgenden Darstellungen der Geschichte der Medienpädagogik als autobiografische Dokumente, als Erzählungen der eigenen Geschichte gelesen. In den Mittelpunkt wird mit der biografischen Methode zunächst die Frage danach, was erzählt wird und die Frage danach, wie die eigene Geschichte erzählt wird, gerückt. Interviews oder Transkriptionen sind dazu nicht erforderlich, da es sich um bereits veröffentlichte Daten handelt.

Da es nur um ein Experiment geht, wird statt einer möglichen Vollerhebung zufällig verfügbares Material herangezogen. Ebenfalls nicht verfolgt wird die Option, eine Langzeitstudie vorzunehmen, mit der die Veränderung der Erzählung von Biografien im Lebenslauf betrachtet wird. Das würde vor allem die von den Herausgeberinnen für diesen Beitrag erwünschte Zeichenzahl überschreiten. Wegen dieser Grenze kann hier die Analyse auch nicht im Detail dargestellt werden. Das ist auch nicht erforderlich - das analysierte Material ist veröffentlicht und muss nicht erst vorgestellt werden. 


\section{Ergebnisse}

Schorb erwähnt zunächst den unterrichtlichen Einsatz von Medien seit den Höhlenmalereien, verbindet den Anfang der Geschichte der Medienpädagogik jedoch mit dem Aufkommen der massenhaften Reproduktion von Schrift und Bild im Zuge der Industrialisierung. Medien «trafen dabei auf Ablehnung» (Schorb 1995, 18). Das ändert sich mit der Reformpädagogik, die eine positive Nutzung, etwa des Films, erreichen will, indem das wahre Bild gezeigt und der Sensationsfilm zensuriert wird. Dabei fanden die Kinoreformer «dort staatliche Anerkennung, wo sie für das System funktional waren» (ebd., 23). Schorb überspringt dann, wie alle anderen Erzählerinnen und Erzähler auch, den ersten Weltkrieg und stellt für die Weimarer Republik fest, dass die Medienpädagogik für den Staat witzlos war. Innerhalb der Medienpädagogik rückt in dieser Zeit der Einzelne und sein Medium in den Mittelpunkt. Auch die Nationalsozialisten brauchten keine medienpädagogische Legitimation für ihren Umgang mit Medien, nutzten aber die Möglichkeiten, z.B. des Unterrichtsfilms, für ihre Zwecke. Gegen diese Tendenzen entwickelt Reichwein, dem es darum ging, «bei den Heranwachsenden selbst die Fähigkeiten zum Sehen zu schulen» (ebd., 30) eine an kritischen Rezipientinnen und Rezipienten orientierte Position. Nach dem zweiten Weltkrieg wurden auch angesichts der neuen Entwicklungen die Debatten fortgeführt - kontrolliert durch einen Grundkonsens. «Große Minderheiten wandten sich gegen die Remilitarisierung und die Ausschaltung von Gesellschaftskritikern - sie blieben Minderheiten» (ebd., 31f.). Zentral wurde die an die Reformpädagogik anschliessende Bewahrpädagogik, die das gute Bewahren und das Schlechte verbieten wolle, was insbesondere auch zum Schulterschluss mit religiösen Interessen führte: Es geht darum, dem Kind «die rechten Werte vorzuhalten» (ebd., 33). In den 6oern wurde kritische Reflexion nach wie vor zurückgedrängt. Zeitgleich entstand ein Markt für Bildungsmedien, was dafür sorgte, dass Medien «nicht mehr als erziehungsabträglich gekennzeichnet» (ebd., 44) wurden. In den 1970er Jahren wurden dann die ersten Professuren für Medienpädagogik geschaffen. Zeitgleich wurde die ideologiekritische Medienpädagogik entwickelt, die allerdings zunächst defensiv blieb - wenn auch aus anderen Gründen als die Bewahrpädagogik. Das ändert sich erst mit technischen Entwicklungen und Theorien, die das handelnde Subjekt in den 
Mittelpunkt stellen (ebd., 47). In den Mittelpunkt rückt die kommunikative Kompetenz. «Die Medienpädagogik der 8oer Jahre suchte sich erstmals [...] gegen die Funktionalisierung durch Politik und Ökonomie zur Wehr zu setzen und eine unabhängige Position einzunehmen» (ebd., 54).

Die Erzählung wird von Schorb so angelegt, dass sie auf die handlungsorientierte Medienpädagogik als Höhepunkt zuläuft - alles Vorherige wird als einseitig und begrenzt dargestellt. Indem der Autor sich als Vertreter einer handlungsorientierten Medienpädagogik zu erkennen gibt, wird er selbst zum Helden seiner Geschichte. Er nimmt die Position des auktorialen Erzählers ein, der vor allem das Dilemma zwischen der positiv-konservativen und negativ-konservativen Position durch eine Selbstüberwindung, hier durch die Entwicklung einer positiv-progressiven Position, löst. Der Wert einer am Individuum orientierten unabhängigen Medienpädagogik wird so mit dem Wert des individuell-unabhängigen Erzählers identifiziert, der seinen Text auch jenseits seiner Funktion als rationalistisches Konstrukt (Foucault 1988) im Griff hat.

Schäfer erzählt die Geschichte der Medienpädagogik als Geschichte der Medien (Schäfer 2001, 27). Der Beginn der Medienpädagogik, die als Teil der Allgemeinen Pädagogik beschrieben wird, wird von ihm mit der Entwicklung der Schriftsprache im alten Griechenland markiert und die Einführung von Buchdruck als wesentlicher Entwicklungsschritt dargestellt.

Insofern die Darstellung eher knapp und ungenau erfolgt, bringt Schäfer zum Ausdruck, dass er die Geschichte der Medienpädagogik nicht als Medienpädagoge erzählt. Er sieht sich im Rahmen der abendländischen Geschichte, die er überblickt und in der es jenseits der Schrift nur unbekanntes Land gibt. Schäfer erzählt als Lehrer, der die Medienpädagogik über die Bedeutung der Medien unterrichtet. Indem Geschichte als durch Medien konstituiert dargestellt wird, wird Medien ein überragender Wert zugewiesen.

Blaschitz und Seibt beginnen ihre Erzählung der Geschichte der österreichischen Medienpädagogik im Jahre 1896 und mit einem Verweis auf die damals verbreitete Diskussion minderwertiger Literatur und Leswut, die eingeschränkt werden sollte, was dann ähnlich auch für das Kino geltend gemacht wurde. Dem stellen Sie das «Bemühen, positive Gegenbeispiele 
zu entwickeln» (Blaschitz und Seibt 2008) gegenüber und betonen, dass ein jeweils neues Medium solange abgelehnt wurde, «bis es in Bildungsmassnahmen eingebunden wurde und somit legitimiert war» (ebd.). Dem folgt eine Darstellung der Verwendung von Medien durch die nationalsozialistische Propaganda. Nach dem zweiten Weltkrieg bis in die 1970er Jahre prägte dann die katholische Kirche die Filmerziehung. Massenmedien wurden problematisiert, allerdings nicht mit den Mitteln der Frankfurter Schule, sondern als Geheime Miterzieher. Fernsehen, so erzählen die Beiden, wurde in Österreich bis in die 1980er Jahre in hohem Masse als Bildungsfernsehen betrieben, dann aber unter dem Einfluss der durch Satelliten- und Kabeltechnik empfangbaren kommerziellen Programme in ein Unterhaltungsbildungsprogramm entwickelt. 2001 sollten dann mit der Computermilliarde Schulen Zugang zum Internet erhalten, während zugleich Computerspiele abgelehnt wurden. Medienbildung wird dabei «von den jeweiligen für Unterricht zuständigen Ministerien unterstützt und geprägt» (ebd.). Daneben gab es ab den 1970er Jahren im Anschluss an die Entwicklungen in Deutschland vor allem in der Jugendsozialarbeit Projekte der aktiven Medienarbeit, die handlungsorientierte und emanzipatorische Ansprüche verfolgten. Zentral blieben aber bewahrpädagogische Ansätze, auch in der Forschung. Um 1980 wurden dann in Universitäten aktive Medienarbeit und Medienmündigkeit in den Mittelpunkt gerückt und mit dem E-Learning die Mediendidaktik präsenter.

Blaschitz und Seibt erzählen als von diversen Spannungen Betroffene. Sie stehen zwischen Schutz und positiver Nutzung, zwischen Jugendsozialarbeit und Schulen und zwischen bewahrpädagogischen und emanzipatorischen Theorien. In den Mittelpunkt rücken sie jedoch eine Haupterzählung, die durch die «positive Nutzung» markiert wird und mit der insbesondere das wertvolle Bildungsfernsehen assoziiert wird, das einsam als guter Kompromiss aus den Spannungen herausragt, leider aber einen frühen Tod gefunden hat - womit die Heldin und der Held tragisch scheitern.

Hüther und Podehl beginnen ihre Erzählung doppelt: einerseits mit der Geschichte der wissenschaftlichen Disziplin, die in den 1960er Jahren etabliert wurde, und andererseits mit der praktischen Medienarbeit, die, wie sie bemerken, erst «nachträglich zu einer Geschichte der Medienpädagogik 
verdichtet» (Hüther und Podehl 2017, 117) wird. Sie betonen, dass Medienpädagogik «in Abhängigkeit von den jeweiligen politischen Gegebenheiten» (ebd., 118) Zielkategorien und Ansätze entwickelt hat und eröffnen mit einer Darstellung einer Bewahrpädagogik, die vor allem von staatlicher Obrigkeit und Klerus ausgeht. In diesen Rahmen werden auch die mit wertvollen Medien vermittelten Wertmassstäbe eingetragen. Der zweite Weltkrieg wird als Zeit der Propaganda gekennzeichnet. Die Zeit zwischen 1945 und den 1960er Jahren wird wie die Zeit vor dem ersten Weltkrieg als präventiv-normative Periode bezeichnet, in der Filmwirkungsforschung und Jugendmedienschutz einflussreich waren und die Selbstbewahrung vor schädlichen Einflüssen in den Mittelpunkt rückt. Das wurde durch jugendliche Protestkulturen - erwähnt wird vor allem der Rock ' $n$ ' Roll - unterlaufen. Ab Mitte der 1960er Jahre wird der sinnvolle Gebrauch der Medien in den Mittelpunkt gerückt und bald zur emanzipatorisch-politischen Medienpädagogik erweitert, die das «Durchschauen kommunikativer Abläufe und Hintergründe» (ebd. 122) in den Mittelpunkt rückt. Das wird dann fliessend in die reflexiv-praktische Medienpädagogik weiterentwickelt: «Diese Medienpädagogik verfolgt das Ziel, Medien auch zur Veränderung von Handeln und Verhalten einzusetzen, indem sie die Möglichkeiten der Erkenntnisgewinnung und Bedürfnisartikulation durch Aktive, sprich reflexiv-praktische Medienarbeit fördert» (ebd, 122). Aktuell steht die $\mathrm{Me}$ dienpädagogik vor allem angesichts der Verbreitung von sozialen Medien vor der Aufgabe, «sinnstiftende Orientierung» (ebd, 124) zu schaffen, in dem Anwendungswissen in Orientierungswissen eingebettet wird. Das wird in Zusammenhang mit der Diskussion des Medienbildungsbegriffs gestellt, dessen «Versatzstücke [...] anders als die digitalen Medien noch auf ihre Vernetzung warten» (ebd., 124).

Auch Hüther und Podehl nehmen die Position von Helden ein, die mit der handlungsorientierten Medienpädagogik einen grossen Sieg errungen haben. Auch kommt in dieser Erzählung ein Wohlwollen gegenüber der jugendlichen Protestkultur der 1960er Jahre zum Ausdruck, nicht aber gegenüber anderen jugendlichen Protestkulturen wie z.B. der Wandervogelbewegung. Das könnte Ausdruck der eigenen Biografie sein, die so in die erzählte Geschichte der Medienpädagogik eingeschrieben wird. Die Position des Helden wird dabei genutzt, um die Verwendung von Medien zur 
Veränderung von Verhalten und Handeln zu legitimieren - die Adressatinnen und Adressaten nach ihren Interessen zu fragen, ist wissenden Helden auch kaum zuzumuten.

Die formulierende Interpretation zeigt, dass die Erzählungen ähnlich gerahmt werden: Es geht zunächst um eine Geschichte der Medien und der Medienindustrie (Moser 2010, 10). Die Geschichte wird dann vor allem thematisch aufgespannt zwischen «schädlichen Einflüssen» und "positiven Möglichkeiten» (Vollbrecht 2001, 25): Die meisten Erzählerinnen und Erzähler erzählen die Geschichte der Medienpädagogik als Überwindung bewahrpädagogischer Ansätze und nationalsozialistischer Propaganda. Diese Überwindung wird mit der Entstehung der Medienpädagogik in den 1960er Jahren verbunden. Dabei wird die Geschichte so erzählt, dass Bewahrpädagogik und Propaganda durch eine ressourcenorientierte, handlungsorientierte, politische oder kritische Position überwunden werden. Interessanterweise wird diese Position dabei nicht expliziert, sondern implizit eingenommen (vgl. etwa die Beiträge in Sander, Gross, und Hugger 2008).

In jedem Fall aber wird die Geschichte der Medienpädagogik als eine Antwort auf gesellschaftliche und technische Entwicklungen erzählt und dabei deutlich, dass alle Erzählerinnen und Erzähler auf den gleichen konjunktiven Erfahrungsraum rekurrieren, in dem nicht digitale Massenmedien dominant waren. Das schliesst die Lokalisierung der Erzählerinnen und Erzähler in einer kapitalistischen Gesellschaft ein. In dieser Hinsicht kommt in den Erzählungen regelmässig die Kritik der Manipulation vor (vgl. etwa die Erzählung in Hüther und Podehl 1990), aber schon die Bürgerinnen- und Bürgermedien werden kaum erwähnt, was ein Ausdruck des Eindrucks der eigenen Biografien sein könnte. Das gilt auch für den Umstand, dass von digitalen Massenmedien nur selten und wenn, dann wie von einem Fremdkörper erzählt wird.

Nun erlaubt es die rekonstruktive Methode nicht in den Blick zu nehmen, was nicht gesagt wurde. Schweigen bedeutet aber nicht Nichts. Das nicht Gesagte in der Interpretation nicht heranzuziehen ist zwar die Absicht des induktiven Vorgehens. Damit wird aber die Reflexion von Interessen und Werten übergangen. So versteht Bohnsack im Anschluss an Mannheim die dokumentarische Methode als praxeologische Wissenssoziologie 
und übernimmt dabei den Begriff der Seinsverbundenheit des Wissens und den Begriff des konjunktiven Erfahrungsraums (Bohnsack 2010, 137). Dabei berücksichtigt er nicht, dass Mannheim 1920 einen Studienaufenthalt in Freiburg absolvierte und dort das Seminar «Phänomenologische Übungen» besuchte, das, wie die zugehörige Vorlesung, von Heidegger gehalten wurde. In der Vorlesung erläuterte Heidegger die These, dass die faktische Lebenserfahrung existenziell zur Philosophie gehöre. Dazu bemerkt Laube: «Mannheim erörtert diesen Zusammenhang in seiner Unterscheidung von «konjunktivem» und «kommunikativem» Denken, die auch «Verbegrifflichungen» in ihrem «existentiellen Bezug» berücksichtigt [...]» (Laube 2000: 282). Nach Laube stammt der Begriff des konjunktiven Denkens aus Heideggers Philosophie, und auch der Begriff der Praxeologie weist eher Bezüge zum Heideggerschen Begriff der faktischen Lebenserfahrung als zum Praxisbegriff von Marx auf, da Bohnsack sich gegen den Ansatz von Marx wendet (Bohnsack 2010, 137).

Dabei geht es hier nicht darum, die Genealogie der dokumentarischen Methode zu diskutieren, sondern lediglich um den Hinweis, dass mit der dokumentarischen Methode wie mit jeder anderen Methode, Interessen (Habermas 2001) zum Ausdruck gebracht werden. Das ist hier wiederum nur relevant, insofern es den Versuch motiviert, das eben Gesagte zu berücksichtigen, in dem das, wovon die dokumentarische Methode schweigt, zum Anlass genommen wird, einen Blick auf das zu werfen, wovon die Erzählerinnen und Erzähler erzählen: Den Wert der Medien.

\section{Diskussion}

In den Erzählungen berücksichtigt wird die Entscheidung für die positiven Möglichkeiten, nicht aber der Erwerb von Eigentum an Medien. Ähnlich wie Theoretikerinnen und Theoretiker der Partizipation vor der Übernahme von Macht zurückschrecken (Swertz 2013), schrecken offenbar Medienpädagoginnen und Medienpädagogen vor der Aufforderung zum Erwerb des Eigentums an Medien zurück. Daran wird sichtbar, dass die eigene gesellschaftliche Position von den Erzählerenden als kritisch beschrieben wird, aber - vermutlich durchaus intendiert - nicht als so kritisch, dass die ökonomischen Bedingungen der Möglichkeit des eigenen Handelns mit 
kritisiert würden. Die Bedingungen der Möglichkeit der Produktion der Erzählungen wird in den Erzählungen zum Ausdruck gebracht, aber nicht distanziert. Das erfordert eine über die reflektierende oder kontextualisierende Interpretation (Friebertshäuser 2016, 26) hinausgehende begriffliche Interpretation.

Einen Ansatzpunkt dafür liefert der Umstand, dass mit der Ablehnung der Ablehnung von Medien in allen Erzählungen der Geschichte der Medienpädagogik zugleich der Wert der Medienpädagogik am Markt (der Ausdruck wird z.B. in Meister 2005 an 118 Stellen verwendet) zum Ausdruck gebracht wurde. So erzählt Schorb, dass die Medienpädagogik immer schon «eine gesellschaftliche Verantwortung» (Schorb 1995, 15) trägt oder Meister, dass die Medienpädagogik an der Produktion der «Werte und Orientierungen» (Meister 1997, 24) beteiligt ist. Das wirft die Frage nach der Schöpfung des Wertes der Medien durch die Medienpädagogik in Märkten auf.

Dafür ist aufschlussreich, dass in medienpädagogischen Veröffentlichungen gelegentlich die Rede davon ist, dass ein didaktischer Mehrwert erreicht werden soll (Lermen 2006), Spiele mit «begründbaren Mehrwerten» (Petko 2008, 2) entwickelt werden sollen und der «Mehrwert des Medieneinsatzes in der Hochschullehre» (Stober 2017) forciert werden soll. Auch wird Medienbildung als «Unterrichtsprinzip mit Mehrwert» (Krucsay 2010) deklariert oder der «Mehrwert» von Learning Analytics argumentiert (Ebner und Ebner 2018), womit die Verwicklung ökonomischer und ethischer Wertschöpfung in der Praxis zum Ausdruck gebracht wird.

Nun erzielen im Kapitalismus weder das Produkt noch die Arbeiterinnen und Arbeitern Gewinn aus Mehrwert, sondern Kapitalistinnen und Kapitalisten. Mehrwert beschreibt Unternehmerinnen- und Unternehmergewinne (Schumpeter 1926), die durch die Ausbeutung von lohnabhängig Tätigen erzielt werden (Marx 1962). Der Mehrwert ist dabei die Differenz des Werts von variablem und konstantem Kapital und dem Wert der Güter. In dem hier zur Rede stehenden Fall sind die Lernenden die produzierten Güter und die Medienpädagoginnen und Medienpädagogen die Arbeiterinnen und Arbeiter. Wenn Medienpädagoginnen und Medienpädagogen in dieser Konstellation eine Steigerung des Mehrwerts fordern, möchten sie mehr ausgebeutet werden. 
Diese Interpretation geht allerdings von der Annahme aus, dass Medienpädagoginnen und Medienpädagogen produktive Arbeit leisten. Wenn aber niemand da ist, für den sich die Arbeit in Kapital verwandelt, dann handelt es sich um unproduktive Arbeit, in der kein Mehrwert erzeugt wird. Genau das ist in der medienpädagogischen Praxis aber die Regel und nicht die Ausnahme. Medienpädagoginnen und Medienpädagogen gehören zur dienenden Klasse und sind Luxusartikel (Marx 1963, 409).

Schorb hat auf dieses Problem mit der Formulierung, dass die «Medienpädagogik [...] für die Administration rein funktional ist» (Schorb 1995, 56), treffend hingewiesen. Mit Marx ist daher zu erwarten, dass Medienpädagoginnen und Medienpädagogen gemeinsame Interessen mit den Kapitalisten entwickeln, was sie tun, indem sie sich zwar kritisch geben, dabei aber Narren (Swertz 2009) bleiben.

In den Blick rückt damit der Gebrauchswert der Medienkompetenzvermittlung für politische Institutionen. Die Notwendigkeit der Medienkompetenzvermittlung wird in den referierten Erzählungen stets mit gesellschaftlichem Bedarf begründet: «Medienpädagogik trägt schon immer eine gesellschaftliche Verantwortung» (Schorb 1995, 15). Politische Institutionen sind zahlende Dienstherren, während die Adressatinnen und Adressaten die Produkte in der Regel kostenlos erhalten, denn was keinen Gebrauchswert hat, ist wertlos.

Der Gebrauchswert für politische Institutionen besteht zunächst darin, dass Medienpädagogik die Verbreitung von Medien in der Gesellschaft legitimiert, indem Sie eine Therapie der Risiken verspricht und Mediennutzung im Allgemeinen protegiert.

An dieser Stelle liegt die Vermutung eines biografischen Bezugs auf der Hand, denn die Forscherinnen und Forscher, die wie ich ihre Arbeit der Medienpädagogik widmen, müssen ihre Geschichte als eine positive Geschichte erzählen, um sich selbst rechtfertigen. Dass das systematisch legitim ist, kann hier zwar rechtfertigend angeführt werden - allerdings nur aus betroffener Perspektive.

Eine Möglichkeit, sich aus diesem Gefängnis zu befreien, ist die Verwendung eines weiten statt eines engen Medienbegriffs, weil Medien damit als Bedingung der Möglichkeit von Pädagogik überhaupt ausgewiesen werden können. Die Beobachtung, dass die Medienpädagogik ein Gebiet 
mit einer eher geringen Zahl an Lehrstühlen ist (Pietraß und Hannawald 2008) legt allerdings nahe, dass es dabei Probleme mit der Differenz zwischen der Innen- und der Aussenperspektive gibt.

Eine weitere Möglichkeit besteht in dem Umstand, dass die Medienpädagogik einen Mehrwert für politische Institutionen erzeugt. Dabei handelt es sich um einen absoluten Mehrwert, weil eine Verbesserung der Produktionsmittel weder in noch von der Medienpädagogik gefordert wird. Es geht auch nicht um die Produkte, sondern um symbolische Werte (Meister u.a. 2008, 23). Während Erziehung von der Zumutung an den Zögling handelt, «zunehmend so zu handeln, als ob er autonom wäre» (Reichenbach 1999), wird von den Adressatinnen und Adressaten der Medienpädagogik nicht verlangt, dass sie so tun können, als ob sie autonom mit Medien umgehen könnten - relevant ist, dass so getan wird, als ob Medienkompetenz vermittelt worden wäre.

Durch die Produktion symbolischer Werte ermöglicht die Medienpädagogik die Akkumulation von symbolischem Kapital, das dann in ökonomisches Kapitel transformiert werden kann. So kann etwa Werbung durch Werbekompetenzvermittlung legitimiert werden, womit Probleme zugleich individualisiert werden. Das gilt auch für politische Werbung, die, wie jede andere Werbung, zwar dem Jugendmedienschutz unterliegt, bei der aber auf eine Alterskennzeichnung verzichtet wird. Der Preis des medienpädagogischen Angebots ist dabei geringer als der Gewinn, der mit Werbung erzielt werden kann. In dieser Struktur kann zumindest eine angemessene Finanzierung für die Medienkompetenzvermittlung begründet werden.

Die dritte Möglichkeit ist es, durch die Erzeugung symbolischen Kapitals im Rahmen unproduktiver Arbeit Werte zu erzeugen und Gewinn zu machen. Wie das zu machen ist, zeigt ein Blick auf Eliteuniversitäten. Diese akquirieren etwa durch Patente zunächst ökonomisches Kapitel. Daraus und damit wird, auch mittels Werbung, symbolisches Kapitel erzeugt, das dann über den Aufbau von Marken (etwa der Marke www.mit.edu, auf die z.B. Kamin und Meister 2015 reagieren) wieder Distinktionsgewinne ermöglicht, das, etwa durch hohe Studiengebühren, wieder in ökonomisches Kapitel transferiert werden kann. In diese Richtung ist ein erheblicher Teil der Mediendidaktikerinnen und Mediendidaktiker gegangen (z.B. www. 
learntec.com), und genau dafür ist es perfekt, so tun zu können, als wäre man erzogen, denn das ist die optimale Qualifikation dafür so zu tun, als würde man den Lernerfolg steigern. Dass dabei mit der Marke zugleich ein Heldenmythos konstruiert wird, ist offensichtlich.

Dabei kann allerdings einer vierten Möglichkeit nicht ausgewichen werden: Medienpädagoginnen und Medienpädagogen müssen sich emanzipieren, indem sie produktive Arbeit leisten, ihre Produkte den Adressatinnen und Adressaten verkaufen und so die Position der Dienenden, oder, mit Nietzsche, die Sklavenmoral (mit der Nietzsche gezeigt hat, wie Marx Analyse in den Dienst der Herren gestellt werden kann) heldenhaft überwinden.

Damit steht die Medienpädagogik zwischen der Skylla der unproduktiven Arbeit mit dem Ideal der Dienerschaft und der Charybdis der produktiven Arbeit mit dem Ideal der Ausbeutung. In diesem Dilemma kann ein bildender Gehalt in der Korrelation zwischen beiden liegen, wenn mit einer List (Swertz 2017b) eine souveräne Position jenseits dieser Struktur eingenommen wird. Das kann durch eine mediendidaktisch effektive reflexiv-handlungsorientierte Medienkompetenzvermittlung oder eine Medienkompetenz vermittelnde bildungstechnologische Medienpädagogik ausgedrückt werden. Das Dilemma so zwischen Inhalt und Methode zu positionieren eröffnet einen Spielraum, mit dem zur Übersetzung zwischen den beiden unvereinbaren Positionen aufgefordert wird. Ein solcher Übersetzungsprozess ist aber nichts anderes als ein Bildungsprozess.

\section{Literatur}

Alheit, Peter, und Heide von Felden. 2009. «Einführung: Was hat lebenslanges Lernen mit Biographieforschung zu tun?»In Lebenslanges Lernen und erziehungswissenschaftliche Biographieforschung, herausgegeben von Peter Alheit und Heide von Felden, 9-17. Wiesbaden: VS Verlag für Sozialwissenschaften. https://doi.org/10.1007/978-3-531-91520-3_1.

Aufenanger, Stefan. 1999. «Medienbiografische Forschung». In Handbuch Biografieforschung, herausgegeben von Heinz-Hermann Krüger und Winfried Marotzki, 487. Wiesbaden: VS Verlag für Sozialwissenschaften.

Baacke, Dieter. 1973. Kommunikation und Kompetenz: Grundlegung einer Didaktik der Kommunikation und ihrer Medien. München: Juventa-Verlag. 
Baacke, Dieter. 1983. «Normalbiographie, Empathie und pädagogische Phantasie». In Beiträge zum 8. Kongress der Deutschen Gesellschaft für Erziehungswissenschaft vom 22.-24. März 1982 in der Universität Regensburg, herausgegeben von Dietrich Benner, Helmut Heid, und Hans Thiersch, 298-306. Zeitschrift für Pädagogik. 18. Beiheft. Weinheim: Beltz.

Baacke, Dieter, Uwe Sander, und Ralf Vollbrecht. 1990. Lebensgeschichten sind $\mathrm{Me}-$ diengeschichten. Medienwelten Jugendlicher, Bd. 2. Opladen: Leske + Budrich.

Barberi, Alessandro. 2000. Clio verwundet. Hayden White, Carlo Ginzburg und das Sprachproblem in der Geschichte. Wien: Turia+Kant.

Blaschitz, Edith, und Martin Seibt. 2008. «Geschichte und Status Quo der Medienbildung in Österreich». In Medienbildung in Österreich. Historische und aktuelle Entwicklungen, theoretische Positionen und Medienpraxis, herausgegeben von Edith Blaschitz und Martin Seibt, 11-25. Berlin: Lit.

Bohnsack, Ralf. 2010. «Praxeologische Wissenssoziologie». In Hauptbegriffe qualitativer Forschung, herausgegeben von Ralf Bohnsack, Winfried Martozki, und Michael Meuser, 3. Auflage, 137-38. Opladen: Leske+Budrich.

Borkert, Maren, Alberto Martín Pérez, Sam Scott, und Carla de Tona. 2006. «Einleitung: Migrationsforschung in Europa (über nationale und akademische Grenzen hinweg) verstehen». Forum Qualitative Sozialforschung 7 (3): 9. https:// doi.org/10.17169/fqs-7.3.132.

Buchen, Sylvia, und Ingeborg Philipper. 2003. «Biographie, Generation, Gender im Hinblick auf die Nutzung neuer Medien: Was bewirken veränderte Lernarrangements in der Schule?» MedienPädagogik: Zeitschrift für Theorie Und Praxis Der Medienbildung 3 (Jahrbuch Medienpädagogik), 123-35. https://doi. org/10.21240/mpaed/retro/2017.07.07.X.

Campbell, Joseph. 1999. Der Heros in tausend Gestalten. Erstveröffentlichung (1949): The Hero with a thousand faces. Princention, NJ: Princeton University Press. Frankfurt am Main und Leipzig: Insel Taschenbuch.

Ebner, Markus, und Martin Ebner. 2018. «Learning Analytics an Schulen - Hintergrund und Beispiele». Medienimpulse 56 (1): 28. https://journals.univie.ac.at/ index.php/mp/article/view/mil19o/1313.

Foucault, Michel. 1988. «Was ist ein Autor?» In, herausgegeben von Michel Foucault, 7-31. Frankfurt am Main: Suhrkamp.

Friebertshäuser, Barbara. 2016. «Fremde und Fremdes forschend verstehen. Herausforderungen qualitativer Forschung». In Von der Bildung zur Medienbildung: Festschrift für Winfried Marotzki, herausgegeben von Dan Verständig, Jens Holze, und Ralf Biermann, 17-42. Medienbildung und Gesellschaft, Band 31. Wiesbaden: Springer VS. https://doi.org/10.1007/978-3-658-10007-0_2.

Fuchs, Thorsten. 2011. Bildung und Biographie: eine Reformulierung der bildungstheoretisch orientierten Biographieforschung. Pädagogik. Bielefeld: Transcript.

Habermas, Jürgen. 2001. Erkenntnis und Interesse. Mit einem neuen Nachwort. 13. Aufl. Frankfurt am Main: Suhrkamp.

Hayek, Friedrich August von. 2005. Die Verfassung der Freiheit. 4. Aufl (1. Aufl 1971, engl. 1960). Tübingen: Mohr Siebeck. 
Hickethier, Knut. 1980. «Kino und Fernsehen in der Erinnerung ihrer Zuschauer». Ästhetik und Kommunikation 42: 53-66.

Hönigswald, Richard. 1926. Vom Problem des Rhythmus. Wiesbaden: Springer Fachmedien.

Hönigswald, Richard. 1927. Über die Grundlagen der Pädagogik. Ein Beitrag zur Frage des pädagogischen Universitäts-Unterrichts. 2. umgearbeitete Auflage. München: Ernst Reinhardt.

Humboldt, Wilhelm von. 1997. «Natur und Beschaffenheit der Sprache überhaupt». In Bildung und Sprache, herausgegeben von Clemens Menze, 5., durchges. Aufl, 89-100. Schöninghs Sammlung pädagogischer Schriften. Paderborn: Schöningh.

Hüther, Jürgen, und Bernd Podehl. 2017. «Geschichte der Medienpädagogik». In Grundbegriffe Medienpädagogik, herausgegeben von Bernd Schorb, Anja Hartung-Griemberg, und Christine Dallmann, 117-24. München: kopaed.

Hüther, Jürgen, und Bernd Reinhard Podehl. 1990. «Geschichte der Medienpädagogik». In Grundbegriffe der Medienpädagogik, herausgegeben von Jürgen Hüther, Bernd Schorb, und Christiane Brehm-Klotz, 2. neubearbeitete Auflage. Medien-Technik-Bildung 1. Ehningen bei Böblingen: Expert Verlag.

Kamin, Anna-Maria, und Dorothee M. Meister. 2015. «Lehren Und Lernen Mit Digitalen Medien in Der Erwachsenen- Und Weiterbildung». In Die Geschichte Der Medienpädagogik, herausgegeben von Dorothee M. Meister, Friderike von Gross, und Uwe Sander, 156-81. https://pub.uni-bielefeld.de/record/2914740.

Krucsay, Susanne. 2010. «Medienbildung - ein Unterrichtsprinzip mit Mehrwert. Versuch einer Annäherung.» Medienimpulse 48 (2): 4. https://journals.univie. ac.at/index.php/mp/article/view/mi223/484.

Laube, Reinhard. 2000. «Mannheims 〈Kategorie der Bürgerlichkeit): Bürgerlichkeit und Antibürgerlichkeit im Spiegel der Suche nach der (wirklichen Wirklichkeit).» In Karl Mannheims Analyse der Moderne. Mannheims erste Frankfurter Vorlesung von 1920. Edition und Studien, herausgegeben von Martin Endreß und Ilja Srubar, 1996:263-92. Jahrbuch für Soziologiegeschichte. Wiesbaden: Springer Fachmedien.

Lermen, Markus. 2006. «Pädagogik Online: Einsatzmöglichkeiten netzbasierter Lehre in der Ausbildung von Lehrerinnen und Lehrern». MedienPädagogik: Zeitschrift für Theorie und Praxis der Medienbildung 10 (Medien in der Erziehungswissenschaft II), 1-16. https://doi.org/10.21240/mpaed/10/2006.04.21.X.

Marx, Karl. 1962. Das Kapital. Band I - Kritik der politischen Ökonomie. Bd. 23. Karl Marx - Friedrich Engels - Werke. Berlin: Dietz Verlag. http://www.mlwerke. $\mathrm{de} / \mathrm{me} / \mathrm{me} 3 / \mathrm{me} 23$ 000.htm.

Marx, Karl. 1963. Das Kapital. Band II. Bd. 24. Karl Marx - Friedrich Engels - Werke. Berlin: Dietz Verlag.

Meder, Norbert. 1986. «Der Sprachspieler - oder Kreativität als Selbstkonzept im Zeitalter der Information». In Kreativität und Leistung. Wege und Irrwege der Selbstverwirklichung, herausgegeben von Konrad Adam, 140-49. Köln: Bachem. 
Meder, Norbert. 2015. «Neue Technologien und Erziehung/Bildung». Medienimpulse 53 (1). https://journals.univie.ac.at/index.php/mp/article/view/mi788.

Meder, Norbert. 2017. «Überlegungen zur Konstitution der Medienpädagogik». MedienPädagogik: Zeitschrift für Theorie Und Praxis Der Medienbildung 29 (Konstitution der Medienpädagogik), 1-16. https://doi.org/10.21240/ mpaed/29/2017.09.01.X.

Meister, Dorothee M. 1997a. «Zur medienbasierten Pluralisierung von Orientierungen». In Orientierung durch Medien-Orientierung trotz Medien, herausgegeben von Klaus Koziol und Gerfried W. Hunold, 19-24. Stuttgart: Fachstelle für Medienarbeit.

Meister, Dorothee M. 1997b. Zwischenwelten der Migration. Biographische Übergänge jugendlicher Aussiedler aus Polen. Weinheim und München: Juventa.

Meister, Dorothee M. 2004. «Trends bei Erwartungen und Ansprüchen an Weiterbildung im Kontext gewandelter Arbeitsverhältnisse.» Report: Zeitschrift für Weiterbildungsforschung 27 (1): 89-95. http://www.die-bonn.de/doks/meister0401.pdf.

Meister, Dorothee M. 2005. «Einflüsse Neuer Medien auf die Weiterbildung: Rahmenbedingungen, System- und Feldadaptation sowie Anforderungen und Potenziale». https://pub.uni-bielefeld.de/record/2302463.

Meister, Dorothee M. 2007. «Lernen, Gesellschaft und Online-Medien. Pädagogische Überlegungen zu biographischen Lerngewohnheiten in Zeiten des Online-Lernens.» In Perspektiven der Bildung. Kinder und Jugendliche in formellen, nicht-formellen und informellen Bildungsprozessen, herausgegeben von Marius Harring, 259-78. Wiesbaden: VS Verl. für Sozialwissenschaften.

Meister, Dorothee M. 2008. «Schule und Medien». In Handbuch der Schulforschung, herausgegeben von Werner Helsper und Jeanette Böhme, 513-29. Wiesbaden: VS Verlag für Sozialwissenschaften. https://doi.org/10.1007/978-3-531-910956_19.

Meister, Dorothee M., Uwe Sander, Klaus Peter Treumann, Eckhard Burkatzki, Jörg Hagedorn, Mareike Strotmann, und Claudia Wegener. 2008. Mediale Gewalt: Ihre Rezeption, Wahrnehmung und Bewertung durch Jugendliche. Wiesbaden: VS Verlag für Sozialwissenschaften. https://doi.org/10.1007/978-3-53190961-5_1.

Meister, Dorothee M., und Bianca Meise. 2010. «Emergenz neuer Lernkulturen Bildungsaneignungsperspektiven im Web 2.0». In Jahrbuch Medienpädagogik 8: Medienkompetenz und Web 2.0, herausgegeben von Bardo Herzig, Dorothee Meister, Heinz Moser, und Horst Niesyto, 183-99. Wiesbaden: VS Verlag für Sozialwissenschaften. https://doi.org/10.1007/978-3-531-92135-8_10.

Metz, Marina. 2016. Migration - Ressourcen - Biographie. Wiesbaden: Springer Fachmedien Wiesbaden. https://doi.org/10.1007/978-3-658-13664-2.

Moser, Heinz. 2010. Einführung in die Medienpädagogik: Aufwachsen im Medienzeitalter. 5., durchgesehene und erweiterte Auflage. Lehrbuch. Wiesbaden: VS, Verlag für Sozialwissenschaften. 
Petko, Dominik. 2008. «Unterrichten mit Computerspielen. Didaktische Potenziale und Ansätze für den gezielten Einsatz in Schule und Ausbildung». MedienPädagogik: Zeitschrift für Theorie und Praxis der Medienbildung 15 (Computerspiele und Videogames), 1-15. https://doi.org/10.21240/mpaed/15_16/2008.11.07.X.

Pietraß, Manuela, und Sebastian Hannawald. 2008. «Der Stand der universitären Medienpädagogik: Professuren, Studiengänge und Studienabschlüsse», 24.

Pöyskö, Anu. 2009. «Medienbiografie - ein Leben voller Medien». Magazin Erwachsenenbildung 6. http://www.erwachsenenbildung.at/magazin/og-6/ mebog-6.pdf.

Rebillot, Paul, und Melissa Kay. 2011. Die Heldenreise. Das Abenteuer der kreativen Selbsterfahrung. Penzing bei Wasserbug am Inn: Eagle Books.

Reichenbach, Roland. 1999. Demokratisches Selbst und dilettantisches Subjekt. Demokratische Bildung und Erziehung in der Spätmoderne. Freiburg im Üechtland: Universität Freiburg.

Rogge, Jan Uwe. 1982. «Die biographische Methode in der Medienforschung». Medien und Erziehung 26: 1982.

Rogge, Jan-Uwe. 1980. «Möglichkeiten des medienbiografischen Ansatzes in der Rezeptionsforschung». Elefantenbaby, Nr. 9: 15-23.

Sander, Uwe, Friederike von Gross, und Kai-Uwe Hugger, Hrsg. 2008. Handbuch Medienpädagogik. 1. Auflage. Wiesbaden: VS, Verlag für Sozialwissenschaften.

Schäfer, Karl-Herrmann. 2001. «Medienpädagogik als Teildisziplin der Allgemeinen Erziehungswissenschaft». In Jahrbuch Medienpädagogik 1, herausgegeben von Stefan Aufenanger, Renate Schulz-Zander, und Dieter Spanhel, 17-45. Wiesbaden: VS Verlag für Sozialwissenschaften. https://doi.org/10.1007/9783-322-97494-5_2.

Schäffer, Burkhard. 2005. «Generationsspezifische Medienpraxiskulturen. Zu einer Typologie des habituellen Handelns mit neuen Medientechnologien in unterschiedlichen Altersgruppen». MedienPädagogik: Zeitschrift für Theorie Und Praxis Der Medienbildung 5 (Jahrbuch Medienpädagogik), 193-215. https://doi. org/10.21240/mpaed/retro/2017.09.10.X.

Schorb, Bernd. 1995. Medienalltag und Handeln. Medienpädagogik in Geschichte, Forschung und Praxis. Opladen: Leske + Budrich.

Schumpeter, Joseph. 1926. Theorie der wirtschaftlichen Entwicklung. Eine Untersuchung über Unternehmergewinn, Kapital, Kredit, Zins und den Konjunkturzyklus. München und Leipzig: Duncker und Humblot.

Seyffer, Walter. 2011. Helden für ein Leben. Die heldenhafte Lebensreise des Menschen nach Josphe Campbell und ihr Einfluss auf den individuellen Lebenslauf. Ein Beitrag zur anthroposopischen Biografiearbeit. Frankfurt am Main: Info3 Verlag.

Stober, Regina. 2017. «Der Mehrwert des Medieneinsatzes in der Hochschullehre am Beispiel Studierenden-erstellter Videos». MedienPädagogik: Zeitschrift für Theorie Und Praxis Der Medienbildung 28 (Bildung gemeinsam verändern), 11622. https://doi.org/10.21240/mpaed/28/2017.03.04.X. 
Swertz, Christian. 2009. «Narren und Könige. Der Gebildete im Spannungsfeld von Wahnsinn und Macht.»In Weitermachen? Einsätze theoretischer Erziehungswissenschaft, herausgegeben von Richard Kubac, Christine Rabl, und Elisabeth Sattler, 152-64. Würzburg: Königshausen und Neumann.

Swertz, Christian. 2013. «Freiheit durch Partizipation. Ein Oxymoron?» In Partizipative Medienkulturen. Positionen und Untersuchungen zu veränderten Formen öffentlicher Teilhabe, herausgegeben von Ralf Biermann, Johannes Fromme, und Dan Verständig, 69-88. Wiesbaden: Springer.

Swertz, Christian. 2017a. «Rhythmuserleben mit Medien. Medienpädagogische Anmerkungen zur Produktion zeitlicher Gliederungen». Medienimpulse 55 (3): 16. https://journals.univie.ac.at/index.php/mp/article/view/mill14/1260.

Swertz, Christian. 2017b. "Orientierungskönnen in der Leonardo-Welt». Erziehungswissenschaft 28 (2): 9-18. https://doi.org/10.3224/ezw.v28i2.2.

Vollbrecht, Ralf. 2001. Einführung in die Medienpädagogik. Weinheim und Basel: Beltz.

Wigger, Lothar. 2016. «Hegels Bildungstheorie und die bildungstheoretische Biografieforschung». In Von der Bildung zur Medienbildung, herausgegeben von Dan Verständig, Jens Holze, und Ralf Biermann, 109-30. Wiesbaden: Springer Fachmedien Wiesbaden. https://doi.org/10.1007/978-3-658-10007-0_6. 